\title{
Surgical treatment of anogenital hemangiomas of infancy
}

\author{
Bulea Raluca1,2, Enescu DM1,2, Giuvelea Steluța2, Stoicescu Simona1,2, Alexandru Raluca², Ioniță D² \\ 1 Plastic and Reconstructive Surgery Chair, Emergency Clinical Hospital for Children "Grigore Alexandrescu", Faculty of Medicine, University of Medicine and \\ Pharmacy "Carol Davila" Bucharest \\ 2 Clinic of Plastic Surgery, Reconstructive microsurgery and Burns, Emergency Clinical Hospital for Children "Grigore Alexandrescu" Bucharest
}

\begin{abstract}
Introduction Infantile hemangiomas $(\mathrm{IHs})$ are the most common benign tumors of the soft tissue in infants and children and they often represent a serious challenge for the treating physician. Hemangiomas located in the anogenital region represent only about $1 \%$ of all $I H s$, but raise special concerns as they have the propensity to ulcerate. This condition may appear spontaneously, or could result from therapeutic procedures. Ulceration is extremely painful and takes many weeks of conservative therapy to heal.

Material and Methods. The aim of this study is to present the surgical approach of the IHs located in the anogenital area and the outcomes of this treatment option.

Results. During a period of 36 months, 11 children (nine girls, two boys) were referred to our plastic surgery department with hemangiomas involving the anogenital, groin and perineum areas. The average follow-up period was of 8 months, during which $82 \%$ of cases experienced complications, especially ulceration. All the target hemangiomas were removed through a lenticular excision and the wound closed with a linear suture.

Conclusions. Our study has shown that surgical excision of a complicated anogenital hemangioma or of a "healthy" hemangioma at high risk for ulceration in the anogenital region is an effective treatment, with fast healing and complete resolution of the pathogenic condition. Lenticular excision and linear closure represent a convenient surgical technique that can be performed as early surgery, during the proliferative stage, or at any time later, when the patient needs treatment, in safety conditions and with good results.
\end{abstract}

Keywords: anogenital hemangiomas, ulceration, infantile hemangiomas, surgical excision.

Received: 15 September 2014 / Accepted: 29 October 2014

\section{Intruduction}

Infantile hemangiomas ( $\mathrm{IHs}$ ) are the most common benign tumors of the soft tissue in infants and children and often represent a serious challenge for the treating physician. IHs have a special evolution in time, divided into three stages. They usually cannot be observed at birth, becoming visible within the first weeks of life, after which they present an explosive growth period, known as the proliferative stage, lasting in most cases up to 9-12 months, less commonly longer [1]. The next period is the involution stage, extending up to the age of 5-7 years, its main feature being a slow regression of the tumor, that may disappear in about $50-70 \%$ of cases [2]. The conclusion of hemangioma's development is the involuted stage, after the age of $10-12$ years, characterized by the presence of sequelae or residuals scars for about half of the patients with this condition [3].

The anatomical distribution of IHs recognizes a high frequency of these vascular tumors in the craniofacial region $(60 \%)$. The next in line is the trunk $(25 \%)$, while the extremities are affected only by $15 \%$ of cases [4]. Hemangiomas located in the anogenital region represent only about $1 \%$ of all IHs [5]. Special sites in this region are perianal area in both sexes and the vulva in girls [6].
The majority of IHs develop without complications. However there is an important group that may present with ulceration and necrosis, bleeding, distortion of involved tissues, obstruction of a vital structure (vision, airways) and congestive heart failure [3]. Ulceration is the most common of all possible IH complications and some anatomic regions have the propensity to ulcerate, especially the face and anogenital area [7]. This condition may appear spontaneously, or may be the result of the therapeutic measures (e.g. laser therapy). Furthermore, ulceration is extremely painful and takes many weeks to heal using the conservative therapies [5].

Management of IHs ranges from observation, topical applications or injections, pharmacologic therapy (systemic corticosteroid, interferon $\alpha-2 \mathrm{a}$, bleomycin, beta blockers, etc.), laser therapy sessions or surgical excision [8]. However, surgical removal of the tumor is the most rapid solution, the time being an important detail for complicated cases, and can provide a convenient functional as well as cosmetic result. The aim of this study is to present the surgical approach of the IHs located in the anogenital area and the outcomes of this treatment option.

\section{Material and Method}

During a period of 36 months, 11 children (nine girls, two boys) were referred to our plastic surgery department with 
hemangiomas involving the anogenital, groin and perineum areas. The average follow-up period was of 8 months after the healing of the surgical wound was complete. The age at the time of surgery varied from 6 weeks to 5 years, with a mean value of 14,5 months.

At the first visit to our clinic all patients were carefully assessed, including medical history, clinical examination and regular laboratory tests. The variables registered included patient's gender, birth-related information (prematurity, when it was the case; birth weight), age at surgery, IH's development stage, anatomical location of the tumor, clinical and morphologic subtype, presence of multiple hemangiomas, tumor size, prior treatment and response to treatment, presence of complications and their onset moment, functional impact, surgical technique, operative and postoperative complications, and reconstructive outcome.

All patients were admitted to the hospital and the tumor excision was performed under general anesthesia. Before surgery, the cases of ulcerated hemangiomas received local treatment, consisting in dressings with antibiotic and/ or corticosteroid ointments and frequent diaper changing. After the tumor removal, patients were administered prophylactic antibiotic in order to prevent any potential infection in this sensible area, treatment for pain and swelling, as well as local cleaning and dressings.

The effectiveness of the treatment was assessed considering the healing of the postoperative wound, the absence of long term complications, the lack of rebound growth of the IH, the scar appearance, the absence of pain and of other functional impairments.

The study was conducted in accordance to the declaration of Helsinki.

\section{Results}

There were 9 girls and 2 boys who presented to our department with IHs of the anogenital region, including perineum and groins. Patient's data are summarized in Table I.
Three of these patients were prematurely born, two of them at 37 weeks, and one at 32 weeks, with very low birth weight, under $1000 \mathrm{~g}$. Average age at the time surgical excision was performed was around 14,5 months, ranging from 6 weeks to 5 years., $64 \%$ of cases $(n=7)$ being treated during the proliferative stage (maximum age 11 months), while the remaining $36 \%(n=4)$ received treatment in the involution stage.

The 11 patients included in this study presented a total of 14 hemangiomas, 11 being localized in our target area, and the other three belonging to other anatomic regions (face, trunk). Most of them (82\%) had a single tumor, and $18 \%$ registered more than one IH (one case had two hemangiomas, and one had three).

Regarding the tumor size and location, the mean diameter was around $3 \mathrm{~cm}$, ranging from $1,5 \mathrm{~cm}$ to $5 \mathrm{~cm}$, and they were affecting three main sites, the labia majora, the buttocks and the upper thigh (with groin involvement in some cases). They were all localized hemangiomas, mostly superficial, but in some cases a deep component could also be noticed, being registered as mixed subtypes. None of the patients presented a purely deep IH.

Complications were registered for 9 out of the 11 patients (82\%), in 5 cases still ongoing at the time of surgery. The most common complication was ulceration, followed by bleeding, pain and interference with urination and dejection. The first moment of complication onset was placed between the age of 1-4 months, in some cases with repeated recurrence, especially of bleeding episodes. This high complication rate and the risk for these complication to perpetuate ware the main indications for surgical treatment. At the same time, it was highly unlikely for the tumors to disappear with no residual marks, due to their thickness and dimensions.

Before referring to our department, the therapeutic management of these IHs was mostly conservative, with either local ointments and dressings (3 cases) or the "wait-and-see" approach (4 cases), and the remaining four cases underwent various laser therapy sessions,

Table I. Patient Data

\begin{tabular}{|c|c|c|c|c|c|c|c|}
\hline $\begin{array}{l}\text { Case } \\
\text { no. }\end{array}$ & Gender & Age (months) & Stage & $\begin{array}{l}\text { No. of } \\
\text { tumors }\end{array}$ & Location & $\begin{array}{l}\text { Presence of } \\
\text { complications }\end{array}$ & Previous treatment \\
\hline 1 & $\mathrm{~F}$ & 1,5 & proliferative & 1 & right buttock & yes & none \\
\hline 2 & $\mathrm{~F}$ & 3 & proliferative & 1 & upper right thigh & yes & none \\
\hline 3 & $\mathrm{~F}$ & 17 & involuting & 2 & upper left thigh & no & none \\
\hline 4 & $\mathrm{~F}$ & 4 & proliferative & 1 & right buttock & yes & local ointments - not specified \\
\hline 5 & $\mathrm{~F}$ & 60 & involuting & 1 & right labia majora & yes & 4 sessions laser $\mathrm{CO}^{2}$ \\
\hline 6 & $\mathrm{~F}$ & 8 & proliferative & 1 & left labia majora & yes & laser NdYAG, 5 sessions \\
\hline 7 & M & 18 & involuting & 1 & left buttock & yes & none \\
\hline 8 & $\mathrm{~F}$ & 5 & proliferative & 3 & upper right thigh & no & laser NdYAG, 3 sessions \\
\hline 9 & $\mathrm{~F}$ & 11 & proliferative & 1 & upper left thigh & yes & laser started at $4 \mathrm{mo}$ \\
\hline 10 & M & 7 & proliferative & 1 & left buttock & yes & local ointments - not specified \\
\hline
\end{tabular}


using $\mathrm{CO}^{2}$ or Nd:YAG lasers. There was no major improvement concerning the size of the IH or the prevention of further complications.

Nine patients present with a Doppler ultrasonography performed at another clinic, and the remaining patients were diagnosed based their history and clinical features.

The surgical excision was performed only for the anogenital hemangiomas, one tumor per patients. In this occasion we did not take any therapeutic action regarding the other secondary non target uncomplicated hemangiomas. Our goal was to remove the entire tumor within one surgery and getting a satisfactory postoperative scar, as small as possible. All the target hemangiomas were removed through a lenticular excision and the wound closed with a linear suture. During the intervention the hemorrhage was minimal, all bleeders encountered were effectively controlled using bipolar diathermy that accomplished the haemostasis. After the surgery, the results were good, with complete wound healing, no perioperative complications and no distortion of normal anatomical landmarks. The follow up period showed complete restoration of physiological functions, no signs of long term complication and no rebound growth of the IHs.

\section{Discussion}

There are little data in the literature concerning the IHs located in the anogenital area. This situation is partly justified by the fact that this specific location is not as frequent as others. They are usually mentioned in the context of ulcerated tumors. However, they raise special concerns to the treating team because their high complication rate and their capacity of causing distress for the child, since the patient is experiencing sleep disturbances and is highly irritable [9]. Girls seem to be more affected by hemangiomas in this area, situation noticed also by other authors [10]. Even though sometimes perineal IHs may be associated with various anorectal, neurologic, renal and genital malformations [5], none of our patients presented such a condition.

Ulceration in the anogenital region, also called "diaper area”, represents a frequent clinical condition. Generally it concerns between $15-25 \%$ of the cases [11], maybe even more in this specific and special region, as seen in our study, thus being the most frequent complication [12]. Ulceration can also lead easily to wound contamination and secondary infection, and the long term outcome is scarring of the involved tissues [10]. It usually occur early during the proliferative phase, around the 4 month of life $[5,7]$ and this was also the case for our patients.

Although the traditional approach for all hemangiomas was mostly conservative, supporting observation, there are authors advocating for an early stage treatment of tumors located in problem zones, as is the anogenital region, to prevent complications [10,13]. Moreover, ul- cerated hemangiomas proved to be the second most frequent condition requiring active treatment [12]. Considering the tumor resection as one active therapeutical measure, we find it justified due to the difficulty of conservative wound management and to the fact that excision leads to alleviation of all accompanying symptoms, provides controlled and minimal scarring and reduction of the tissue loss that is associated with ulcerated and infected hemangiomas [10]. The pulsed dye laser represents an active treatment approach for superficial hemangiomas that do not exceed $2 \mathrm{~mm}$ in depth, with best results in the firs weeks of development [14]. The tumors we resected were mixed or superficial, but had greater thickness.

Surgical excision has the advantages of insuring faster healing than after other treatment methods (1-2 weeks, instead of 1-2 months for systemic corticosteroids [14] or pulsed dye laser which requires several treatment sessions, every 3-4 weeks [16]), improvement of the physiological functions affected by the ulcerated hemangioma and controlled scaring. Due to the hemangiomas' shape and location we perfomed a lenticular excision and direct closure afterwords, with no need for further surgical correction. Another technique that ensures a small scar and good long term results is the circular excision and purse string closure [15], useful especially for round tumors located in visible areas. It has the disadvantage of a longer healing time (3-4 weeks [17]) and sometimes needs another surgery in order to improve the scar appearance by lenticular excision of the first postoperative scar. For some large tumors, serial excisions may be required in order to achieve a satisfactory cosmetic outcome, but it was not the case in our study. The excision techniques, especially the help of the surgical instruments that cauterize while cutting, have the purpose to limit blood losses and to offer a satisfactory postoperative scar.

\section{Conclusions}

Despite not being the most frequent of all IHs, the anogenital hemangiomas deserve a special attention due to the particular features of that area. They have a high risk of developing complications in the proliferative stage, especially painful slow-healing ulcerations. This situation has a negative impact on the patient's capacity to feed and sleep properly, and the treating physician needs to take action. Our study has shown that surgical excision of a complicated anogenital hemangioma or of a "healthy" hemangioma at high risk of becoming ulcerated in the anogenital region is an effective treatment, with fast healing and complete resolution of the pathogenic condition. Lenticular excision and linear closure represent a convenient surgical technique. It can be performed as early surgery, during the proliferative stage, or at any time later, when the patient needs treatment, with good safe results. 


\section{References}

1. Brandling-Bennett H. A., Metry D. W., Baselga E, et al. Infantile Hemangiomas With Unusually Prolonged Growth Phase, Arch Dermatol, 2008,144(12):1632-1637.

2. Marler, J.J., Mulliken, J. B. Vascular Anomalies, in Mathes S. J., Hentz V. R. (eds.): Plastic Surgery,2nd Edition, Volume V: Tumors of the Head, Neck and Skin, Saunders - Elsevier, Philadelphia, 2006, 19-62.

3. Mulliken, J. B. Vascular Anomalies. Infantile Hemangioma, in Thorne C. B., Bartlett, S.P., Beasley R.W, et al (eds.): Grabb and Smith's Plastic Surgery, Sixth edition, Lippincott Williams \& Wilkins, Philadelphia, 2007,191-195.

4. Finn MC, Glowacki J, Mulliken JB. Congenital vascular lesions: clinical application of a new classification. J Pediatr Surg 1983;18:894-900.

5. Grantzow, R, Schäffer, K. Anogenital Hemangiomas, in Mattassi, R, Loose D.A, Vaghi, M (eds.): Hemangiomas and Vascular Malformations, Springer Verlag Italia, 2009, 93-96.

6. Atherton, D.J. Infantile Hemangiomas, Early Hum Dev, 2006;82:789-795.

7. Léauté-Labrèze, C, Prey, S, Ezzedine, K. Infantile Hemangioma: Part II. Risks, complication and treatment, Journal of the European Academy of Dermatology and Venereology 2011; 25(11):1254-1260.

8. Antaya, RJ. Infantile Hemangiomas, at http://emedicine.medscape.com/ article/1083849-overview, updated Jan 2012.

9. Metry, D.W, Hebert, A.A. Benign Cutaneous Vascular Tumors of Infancy, Arch Dermatol 2000;136:905-914.
10. Achauer, B.M., Vander Kam V.M. Ulcerated Anogenital Hemangioma of Infancy, Plast Reconstr Surg 1991,87:861-866

11. Maguiness, S.M, Frieden, I.J. Current Management of Infantile Hemangioma, Semin Cutan Med Surg 2010,29:106-114.

12. Haggstrom AN, Drolet BA, Baselga E, Chamlin, S.L, et al. Prospective Study of Infantile Hemangiomas: Clinical Characteristics Predicting Complications and Treatment, Pediatrics. 2006;118(3):882-887.

13. Berlien H.P. Principles of Therapy of Infantile Hemangiomas and Other Congenital Vascular Tumors of the Newborns and Infants, in Mattassi, R, Loose D.A, Vaghi, M (eds.): Hemangiomas and Vascular Malformations, Springer Verlag Italia, 2009, 49-56.

14. Berlien HP, Laser Therapy of Infantile Hemangiomas and Other Congenita Vascular Tumors of Newborns and Infants, in Matassi, R., Loose, D.A., Vaghi, M. (eds), Hemangiomas and Vascular Malformations, Springer Verlag Italia 2009, 65-77.

15. Pandey A, Gangopadhyay AN, Sharma SP, Kumar V, Gopal SC, Gupta DK. - Conservative management of ulcerated haemangioma - twenty years experience. Int Wound J 2009; 6:59-62.

16. Poetke M, Philipp C, Berlien HP. Flashlamp pumped pulsed dye laser for hemangiomas in infancy. Arch Dermatol 2000;136:628-632.

17. Mulliken JB, Rogers GF, Marler JJ. Circular excision of hemangioma and purse-string closure: the smallest possible scar. Plast Reconstr Surg 2002;109:1544-1554 